\begin{tabular}{c} 
International Journal of Engineering \& Technology, $7(3.6)(2018) 270-275$ \\
International Journal of Engineering \& Technology \\
Website: $w$ ww.sciencepubco.com/index.php/IJET \\
Research paper \\
\hline
\end{tabular}

\title{
Fusion Based Watermarking Scheme with Multi Level DWT \& SVD
}

\author{
Ch. Hima Bindu ${ }^{1 *}$, Maruturi Haribabu ${ }^{2}$, K. Veera Swamy ${ }^{3}$ \\ ${ }^{I}$ IEEE Member, ACM Member, Professor, Department Of ECE, QISCET, Ongole, India. \\ ${ }^{2}$ Asst.Prof., QISCET, Ongole, India. E-Mail:Haribabu.Maruturi@Gmail.Com \\ ${ }^{3}$ Professor, Department Of ECE, QISCET, Ongole, India.E-Mail:Kilarivs@Yahoo.Com \\ *Corresponding Author E-Mail:Hb.Muvvala@Gmail.Com
}

\begin{abstract}
This paper proposes "Fusion based watermarking with multi level DWT \& singular value decomposition" has been implemented. In watermarking scheme, maintaining security and robustness is major hurdle. To address this issue we proposed a novel non blind embedding scheme with Discrete Wavelet transform (DWT) and Singular Value Decomposition (SVD) techniques. This paper details the design of the proposed watermarking scheme and analyses its robustness in the presence of various possible security attacks that involves in degrading the quality of watermark. In the beginning, the cover image color component (especially Red Component) is decomposed into LL, LH, HL, HH with 2 level DWT: these LH \& HL coefficients are further divided into $8 * 8$ blocks and each block is compared to build a fused coefficient. Apply SVD on fused coefficient of cover image and watermark image to embed the singular values with sigmod scaling factor. Finally the watermarked image is generated, after applying inverse SVD \& 2 level DWT. At receiver side the inverse process has been implemented to extract watermark image. The efficiency and performance of the proposed method is verified with Peak Signal to Noise Ratio (PSNR), Root mean square error (RMSE) and Mean Square error (MSE) and compared with recent works of santhi [12] and harsha [13].
\end{abstract}

Keywords: Digital image watermarking, discrete wavelet transform, singular value decomposition, sigmond function.

\section{Introduction}

Image watermarking scheme ensures security to share the data information in different forms like images, audio, \& video. The problem of maintaining security and authentication of multimedia information against various forms of forgery, creating a new platform for the design of novel watermarking schemes [1]. Recently the color image watermarking plays a crucial role in multimedia applications. The color image watermarking has various advantages over gray level images like to secure the higher amount of data and to improve the capacity and visibility. These techniques are well addressed in [2-3] technical papers. The watermarking algorithms are defined into 2 types: spatial domain and frequency domain. In former case the data is embedded directly by modifying pixel values of the host image. In later schemes embed the data by modifying coefficients instead of pixels.

M. Reza, et.al, [4], proposed Robust Dynamic Block-Based Image Watermarking in DWT Domain. In this work they proposed dynamic blocking instead of static blocking for embedding watermark bits. The final experimental results show the high strength and good transparency. Baisa L, et.al, [5] proposed DWT \& SVD Performance Analysis Based Color Image Watermarking Technique with various color model Spaces. This work addressed non blind image watermarking scheme. This scheme tested for different attacks like scaling, rotation etc. M.M. sathik, et.al, [6] worked on DWT based invisible watermarking technique for digital images. This technique deals with the reveal of the watermark data in the absence of original image. Md. M. Rahman, [7] proposed transform domain based Technique to protect the piracy that ensures good visibility and more robustness against attacks.

The following sections are addressed in this way: In Section II, we briefly describe the preliminaries of basics. With this preparation, we propose a new proposed digital watermarking method in Section III. Experimental results are presented in Section IV, and conclusions are given in Section V.

\section{Preliminaries}

This section discuss about basic preliminaries required for the execution of the proposed work:

\section{Discrete Wavelet Transform}

Wavelets are classified into two : (a) orthogonal (where both the filters have identical length) and (b) biorthogonal (where both the filters do not have identical length). Based on the application, either of them can be used. Consider the image $f(x, y)$, then the wavelet decomposition [15] is represented as follows:

Where the wavelet coefficients are

$$
W_{\varphi}\left(j_{0}, m, n\right)=\frac{1}{\sqrt{M N}} \sum_{x=0}^{M-1} \sum_{y=0}^{N-1} f(x, y) \varphi_{j_{0}, m, n}(x, y)
$$




$$
\begin{aligned}
& W_{\varphi}^{i}(j, m, n)=\frac{1}{\sqrt{M N}} \sum_{x=0}^{M-1} \sum_{y=0}^{N-1} f(x, y) \varphi_{j, m, n}^{i}(x, y) \\
& i=\{H, V, D\}
\end{aligned}
$$

Figure 1 shows the representation of wavelet coefficients equation $1-2$.

The Inverse Discrete wavelet transform is defined as:

$$
\begin{aligned}
& f(x, y)=\frac{1}{\sqrt{M N}} \sum_{m} \sum_{n} W_{\varphi}\left(j_{0}, m, n\right) \varphi_{j 0, m, n}(x, y)+ \\
& \frac{1}{\sqrt{M N}} \sum_{i=H, V, D} \sum_{j=j_{0}}^{\infty} \sum_{m} \sum_{n} W_{\varphi}^{i}(j, m, n) \varphi_{j, m, n}^{i}(x, y)
\end{aligned}
$$

The decomposition of 1-D dwt is shown below as:

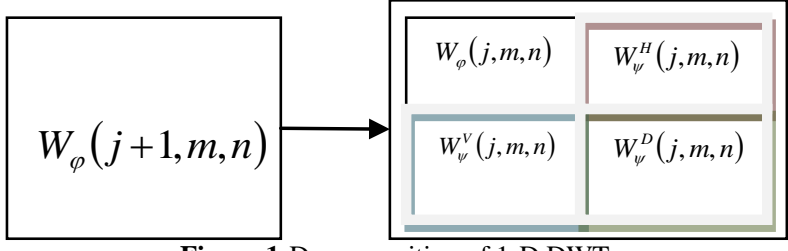

Figure 1:Decomposition of 1-D DWT

For 2-D DWT apply initially one dimensional DWT on two parameters of the data by distinctly filtering and down sampling. In the filter theory, these four sub images resemble to the outputs of high-high $(\mathrm{HH})$, high-low (HL), low-high (LH), and low-low (LL) bands [7, 8, 9]. Later apply 1-D DWT on the LL band, it further decomposed into 4 more sub bands $\mathrm{LL}_{1}, \mathrm{LH}_{1}, \mathrm{HL}_{1}, \mathrm{HH}_{1}$. The complete decimation is shown in figure 2 .

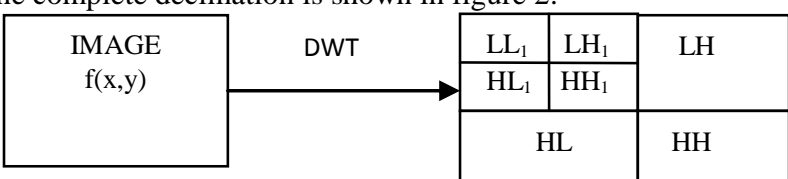

Figure 2: 2-D DWT structure with labeled sub bands

\section{Singular Value Decomposition}

SVD is a one of the famous image watermarking techniques to improve the security. This transform has been used to extract the statistical properties of the image. Let us consider the image $\mathrm{f}(\mathrm{x}, \mathrm{y})$ , SVD of $f$ generates two singular matrices $U$, and $V$ and one diagonal matrix $S$. The advantages of the SVD over other techniques is its stability and human visualization $[10-11,16]$.

$S V D(f(x, y))=U S V^{T}$

For a $N \times N$ square matrix $\mathrm{f}(\mathrm{x}, \mathrm{y})$, its $\mathrm{SVD}$ is represented by Equation. (4). The following equations (5-7) represents the singular matrices $U$, and $V$ and one diagonal matrix $S$.
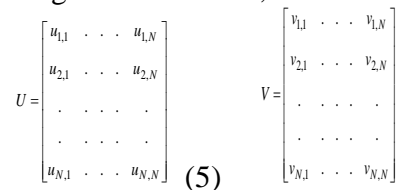

(6)



\section{Proposed Work}

This paper has been proposed on novel non blind embedding scheme with fused coefficient matrix and sigmod function as scaling factor. The main subject of this work is to embed the watermark image data into newly generated cover image data with the help of scaling factor. The purpose of the this embedding algorithm is it must be perceptible and should poses high robustness. This section deals with proposed watermark embedding and extraction with and without attacks. The flow of proposed embedding and extraction is shown in figure $3(\mathrm{a} \& \mathrm{~b})$.

\section{Watermark Embedding Process}

Step 1: Read the color cover image (RGB) \& Gray level watermark image $f \& W$ respectively.

Step 2: Apply two-level DWT on R component of color cover image $f_{R}$. The coefficients are shown below:

$$
\begin{aligned}
& 2-D D W T\left(f^{\operatorname{Re} d}\right) \in\left\{1-D D W T\left(f_{L L}\right), f_{L H} f_{H L}, f_{H H}\right\} \\
& 1-D D W T\left(f_{L L}\right) \in\left\{f_{L L 1}, f_{L H 1} f_{H L 1}, f_{H H 1}\right\}
\end{aligned}
$$

Here $f_{L L}, f_{L H} f_{H L}, f_{H H}$ are 1-level coefficients. The $f_{L L}$ further decomposed for 2-level coefficients: $f_{L L 1}, f_{L H 1} f_{H L 1}, f_{H H 1}$.

Step 3: Apply $8 * 8$ block processing on $f_{L H 1}, f_{H L 1}$ coefficients. Each block of these coefficients are compared with following equation to generate new fused coefficient $(\mathrm{O})$.

$O= \begin{cases}f_{L H 1} & f_{L H 1}>=f_{H L 1} \\ f_{H L 1} & f_{L H 1}<f_{H L 1}\end{cases}$

Step 4: Generate singular values of the fused coefficients $(\mathrm{O})$ and watermark image (W) with SVD transform.

$\left[\begin{array}{lll}u & s & v\end{array}\right]=\operatorname{svd}(O)$

$\left[\begin{array}{lll}u w & s w & v w\end{array}\right]=s v d(W)$

Step 5: Apply embedding process on singular values by using the following equation

$s e=s+\left(\alpha \cdot{ }^{*} s w\right)$

Where $\alpha$ is a scaling factor. It is defined with sigmod function and details are given below:

mean $=\frac{1}{m \times n} \sum_{i=1}^{m} \sum_{j=1}^{n} O(i, j)$

$\alpha 1=\frac{1}{1+e^{-m e a n}}$

$\alpha 1$ (Sigmoid function). This can be used as an activation function. The purpose is to compute the different layer outputs. Finally $\alpha$ is:

$\alpha=\alpha 1 \times \frac{1}{10^{2}}$

This value of $\alpha$ can be varaible to make the watermarking image either visible or nonvisible.

Step 6: Apply Inverse SVD with new embedded singular values:

$O e=u^{*} s e^{*} v^{\prime}$

Step 7: Apply inverse 2 levels DWT to get new Red component of input image and combined with Green \& Blue component to obtain watermarked image $(W D)$. The new Red component is discussed bellow:

$$
\begin{aligned}
& 2 D I D W T\left\{f_{L L}, f_{L H} f_{H L}, f_{H H}\right\} \in w d^{\operatorname{Re} d} \\
& 1 D D W T\left\{f_{L L 1}, O e, f_{H L 1}, f_{H H 1}\right\} \in f_{L L}
\end{aligned}
$$

The final watermarked color image WD and some of the features of cover and watermark image (Note: In this work singular values of cover image 's' from equation (11) and watermark image uw, 
vw from equation (12) are carried to the destination.

Note: A \& B are the identification of the watermark embedding process.

\section{Watermark Extraction Process}

Step 1: Read the watermarked color image $W D$ and some of the features of cover and watermark images to extract the watermark image.

Step 2: Apply two-level DWT on R component ( $w d^{\mathrm{Re} d}$ ) of color watermarked image $W D$. The decomposed sub bands are given below:

$$
\begin{aligned}
& 2 D D W T\left(w d^{\mathrm{Red}}\right) \in\left\{1 D D W T\left(w d_{L}\right), w d_{L H}, w d_{H L}, w d_{H H}\right\} \\
& I D D W T\left(w d_{L L}\right) \in\left\{w d_{L L 1}, w d_{L H 1}, w d_{H L 1}, w d_{H H 1}\right\}
\end{aligned}
$$

Step 3: The $w d_{L H 1} \& w d_{H L 1}$ coefficients are divided into $8 * 8$ blocks and these blocks are used to obtain fused image using following comparison:

$$
O 1= \begin{cases}w d_{L H 1} & w d_{L H 1}>=w d_{H L 1} \\ w d_{H L 1} & w d_{L H 1}<w d_{H L 1}\end{cases}
$$

Step 4: Apply SVD to acquire singular values of fused image O1.

$$
\left[\begin{array}{lll}
u u & s u & v u
\end{array}\right]=s v d(O 1)
$$

Step 5: Now it's time to extract the watermark image by considering carried cover image singular values ' $\mathrm{s}$ ' and watermarked singular values 'su'. By using the given equation the watermark singular values are extracted.

$$
w e 1=\frac{(s u-s)}{\alpha}
$$

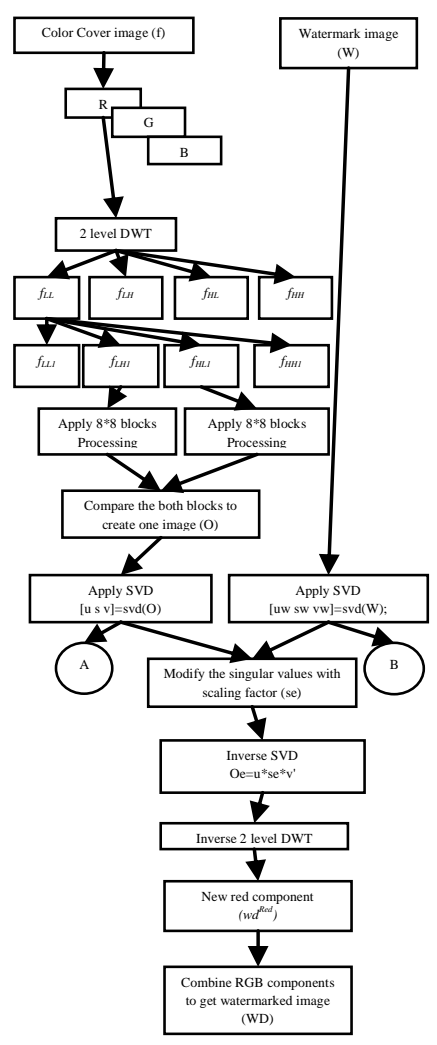

(a)

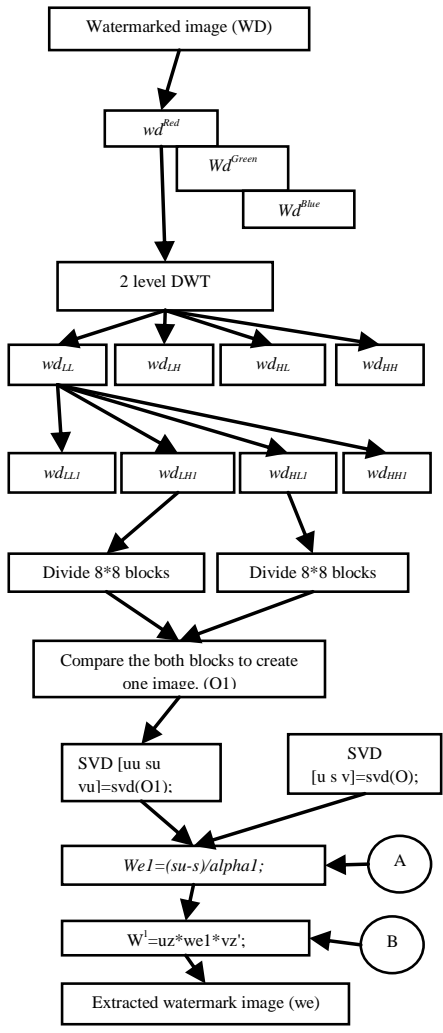

(b)

Figure $3(\mathbf{a} \& \mathbf{b})$ : Flow chart of proposed watermark embedding \& extraction process

Step 6: Apply Inverse SVD to obtain extracted watermark image $\left(w^{1}\right)$.

\section{Results \& Measures}

The proposed scheme simulated in MATLAB. The algorithm operated on various color and gray level images. The cover images, watermark embedding \& extraction images are displayed in Figure 4 without Attacks and Figure 5 is with attacks. The performance measures of these images are tabulated in table $1 \& 2$ with and without attacks respectively. The results of proposed method are compared with earlier work of the paper author [Ref 13] and with existing techniques [Ref 12]. The graphical comparison shown in figure 6 .

$$
w^{1}=u z^{*} w e{ }^{*} v z^{\prime}
$$







(a)

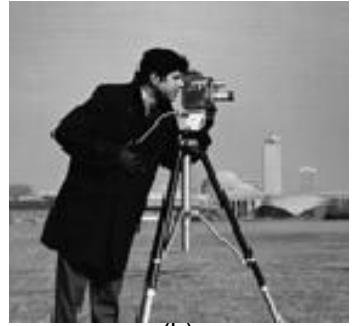

(b)

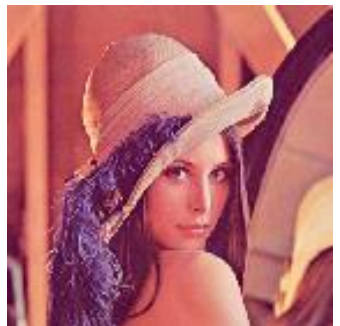

(c)

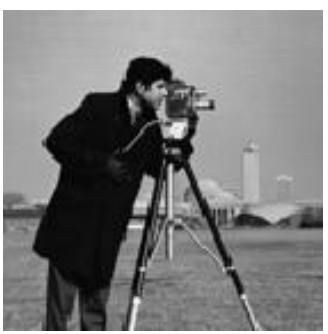

(d)

Figure 4: (a) \& (b) are host and watermark images, (c) \& (d) are watermarked \& extraction images using proposed method

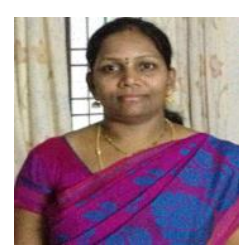

(a1)

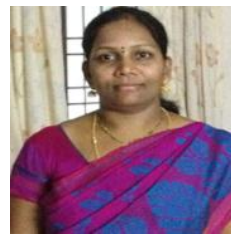

(d1)

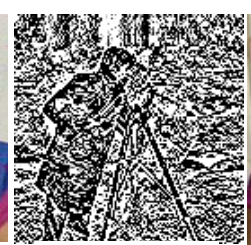

(a2)

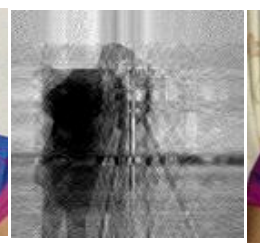

(d2)

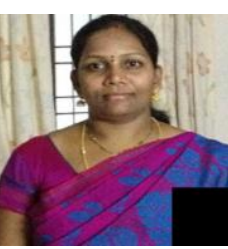

(b1)



(b2)

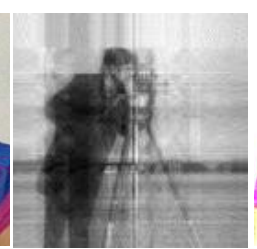

(e2) (c1)

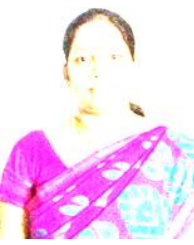

(f1)

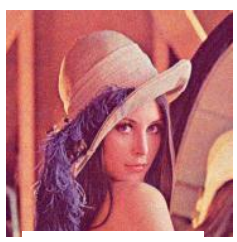

(i1)

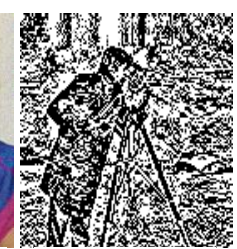

(c2)

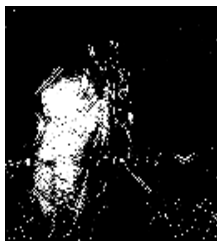

(f2)

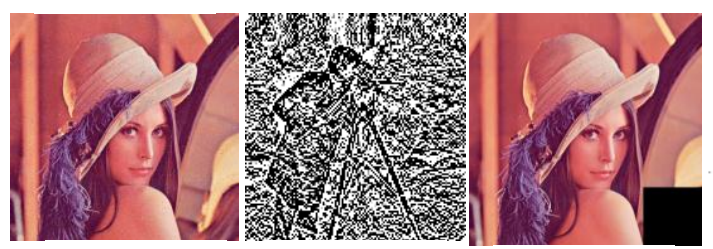

(g1)



(j1) (g2)

(h1)

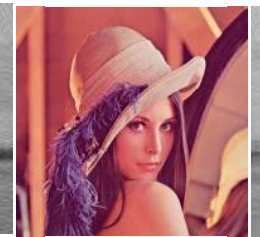

(k1)

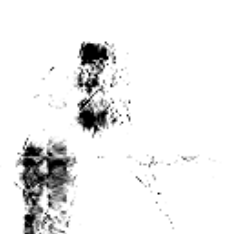

(h2)

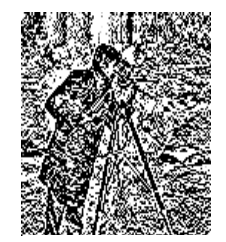

(i2)



(I1)

Figure 5: Proposed method with attacks: watermarked \& extracted watermark images (a1-a2, g1-g2) salt \& pepper noise 0.01, (b1-b2,h1-h2) crop (384:512,384:512), (c1-c2, i1-i2) Gaussian noise 0.01, (d1-d2, j1-j2) brightness, (e1-e2, k1-k2) Darkness, (f1-f2, 11-12) contrast. 

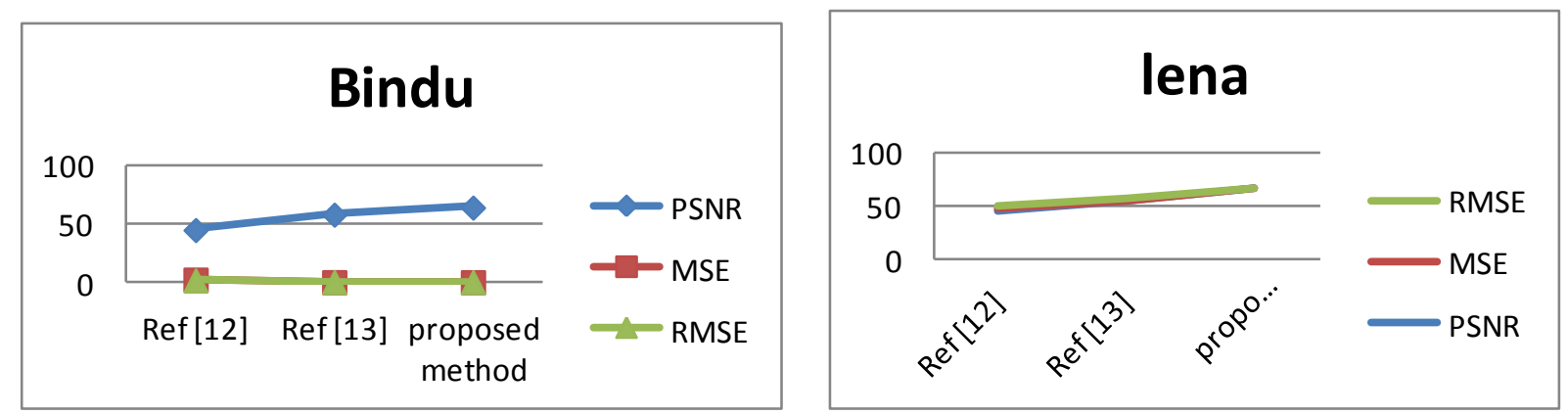

Figure 6: Graphical representation of performances in terms of PSNR, MSE, \& RMSE without attacks

Table 1: Comparison of Measures: PSNR, MSE, \& RMSE with Attacks

\begin{tabular}{|c|c|c|c|c|c|c|c|c|c|c|}
\hline \multirow[t]{3}{*}{ Attacks } & \multirow[t]{3}{*}{ Density } & \multicolumn{9}{|c|}{ Proposed method } \\
\hline & & \multicolumn{3}{|l|}{ PSNR } & \multicolumn{3}{|l|}{ MSE } & \multicolumn{3}{|l|}{ RMSE } \\
\hline & & Lena & Air & Bindu & Lena & Air & Bindu & Lena & Air & Bindu \\
\hline \multirow{3}{*}{$\begin{array}{l}\text { Salt \& } \\
\text { pepper }\end{array}$} & 0.01 & 24.874 & 24.813 & 25.1729 & 195.4711 & 215.02 & 201.5685 & 13.981 & 14.6637 & 14.1975 \\
\hline & 0.1 & 14.829 & 14.963 & 15.0979 & $1.9765 e+003$ & $2.11 \mathrm{e}+003$ & $2.0426 \mathrm{e}+003$ & 44.458 & 45.9956 & 45.1955 \\
\hline & 0.5 & 7.8157 & 7.9596 & 8.10 & $9.8784 \mathrm{e}+003$ & $1.0578 \mathrm{e}+004$ & $1.0228 \mathrm{e}+004$ & 99.389 & 102.8486 & 101.1329 \\
\hline Crop & $(384: 512,384: 512)$ & 15.052 & 15.477 & 19.17 & $1.1614 \mathrm{e}+003$ & $2.01 \mathrm{e}+003$ & 732.9630 & 34.078 & 44.8372 & 27.0733 \\
\hline \multirow[t]{3}{*}{ Gaussian } & 0.01 & 20.385 & 20.164 & 20.19 & 619.7120 & 621.11 & 608.1460 & 24.894 & 24.9223 & 24.6606 \\
\hline & 0.1 & 11.799 & 11.752 & 11.56 & $4.5982 \mathrm{e}+003$ & $4.28 \mathrm{e}+003$ & $4.4917 \mathrm{e}+003$ & 67.810 & 65.4604 & 67.0203 \\
\hline & 0.5 & 7.8128 & 7.8189 & 7.84 & $1.0654 \mathrm{e}+004$ & $1.07 \mathrm{e}+004$ & $1.0730 \mathrm{e}+004$ & 103.21 & 103.5452 & 103.5851 \\
\hline \multirow[t]{3}{*}{ Brightness } & 5 & 34.157 & 34.146 & 34.25 & 24.9876 & 25.0098 & 24.3581 & 4.9988 & 5.0010 & 4.9354 \\
\hline & 10 & 28.160 & 28.129 & 28.25 & 99.7715 & 100.0098 & 97.0507 & 9.9886 & 10.0005 & 9.8514 \\
\hline & 20 & 22.259 & 22.109 & 22.25 & 395.4941 & 400.0098 & 387.1456 & 19.887 & 20.0002 & 19.6760 \\
\hline \multirow[t]{2}{*}{ Darkness } & 5 & 34.148 & 34.146 & 34.14 & 25.0054 & 24.9927 & 24.9675 & 5.0005 & 4.9993 & 4.9967 \\
\hline & 10 & 28.130 & 28.129 & 28.12 & 99.9324 & 99.9231 & 99.4037 & 9.9966 & 9.9962 & 9.9701 \\
\hline Contrast & 5 & 9.1014 & 9.2513 & 6.71 & $1.69 \mathrm{e}+004$ & $6.79 e+03$ & $1.5 \mathrm{e}+004$ & 130.00 & 82.431 & 122.6 \\
\hline
\end{tabular}

Table 2: Comparison of Measures: PSNR, MSE, \& RMSE without attacks.

\begin{tabular}{|l|l|l|l|l|}
\hline Images & Methods & PSNR & MSE \\
\hline Bindu & Ref [12] & 44.5275 & 2.2126 \\
& Ref [13] & 57.6722 & 0.0370 \\
& Proposed method & $\mathbf{6 3 . 6 9 3 7}$ & 1.5141 \\
& Ref [12] & 45.0755 & 0.1925 \\
& Ref [13] & 54.6211 & $\mathbf{0 . 0 0 9 3}$ \\
\hline Air & Proposed method & $\mathbf{6 4 . 9 4 2 8}$ & 2.0208 \\
& Ref [12] & 42.6618 & 0.420 \\
& Ref [13] & 55.6123 & $\mathbf{0 . 0 0 6 9}$ \\
& Proposed method & $\mathbf{6 3 . 2 4 1 4}$ & 3.5229 & 0.1925 \\
\end{tabular}

In Ref 12, the authors worked on hadamard transform with sigmod function as scaling value. The hadamard coefficients had embedded with an adaptively generated scaling factor. In Ref 13, the DWT coefficients of cover and watermark images are embedded with a constant value.

\section{PSNR}

The PSNR is basic measure to check the similarity between cover image (f) verses watermarked image (wd) for human perception. This factor's high value decides the best techniques [14].

$$
\operatorname{PSNR}(d B)=10 * \log _{10}\left[\frac{(255)^{2}}{\frac{1}{M \times N} \sum_{m=0}^{M-1} \sum_{n=0}^{N-1}(f(m, n)-w d(m, n))^{2}}\right]
$$




$$
M S E=\frac{1}{m \times n} \sum_{i=1}^{m} \sum_{j=1}^{n}[f(i, j)-w d(i, j)]^{2}
$$

Where $f(i, j)$ : host image, $y(i, j)$ : watermark image [14].

\section{RMSE}

The RMSE for the watermarked image $w d$ and input image $f$ (both of size $M \times N$ ) are defined as follows.

$R M S E=\sqrt{\frac{1}{M \times N} \sum_{m=1}^{M} \sum_{n=1}^{N}[f(m, n)-w d(m, n)]^{2}}$

Where $f(m, n)$ and $w d(m, n)$ are the pixel value at position $(m, n)$ of $f$ and $w d$ respectively. Smaller the values mean the better image quality.

\section{Conclusion}

The proposed work assisted in preserving secured data transmission and preventing hacking. This paper proposed novel embedding and extraction schemes with fusion and sigmod scaling factors. The performance factors: PSNR, MSE \& RMSE values are $(63.6937,0.0093 \& 0.0962$ for Lena image), $(64.9428$ $0.0069,0.0833$ for air image) \& $(63.2414,0.0103,0.1014$ for Bindu image) without attacks. With this it is concluded that the proposed watermarking schemes shown improvement over the existed methods based on the PSNR \& MSE values. Future work will be focused on extending the algorithm using advanced transformation techniques and adaptable scaling factors to improve reliability.

\section{Acknowledgement}

The authors thank to QIS management for their support.

\section{References}

[1] Seema SS, "DWT-SVD based efficient image watermarking Algorithm to Achieve high robustness and perceptual quality", International Journal of Advanced Research in Computer Science and Software Engineering, Vol.2, No.4,(2012), pp.75-78.

[2] Su Q, Niu Y, Wang G, Jia S \& Yue J, "Color image blind watermarking scheme based on QR decomposition", Signal Processing, Vol.94, (2014), pp.219-235.

[3] Muselet D \& Trémeau A, "Recent trends in color image watermarking", Journal of Imaging Science and Technology, Vol.53, No.1,(2009), pp.10201-1.

[4] Keyvanpour MR \& Merrikh-Bayat F, "Robust dynamic blockbased image watermarking in DWT domain", Procedia computer science, Vol.3, (2011), pp.238-242.

[5] Gunjal BL \& Mali SN, "Comparative performance analysis of DWT-SVD based color image watermarking technique in YUV, RGB and YIQ color spaces", International Journal of Computer Theory and Engineering, Vol.3, No.6,(2011), pp.714-719.

[6] Sathik MM \& Sujatha SS, "A Novel DWT Based Invisible Watermarking Technique for Digital Images", Int. Arab J. $e$ Technol., Vol.2, No.3,(2012), pp.167-173.

[7] Rahman M, "A DWT, DCT \& SVD based watermarking Technique to protect the image piracy", IJMPICT, Vol.4, No.2, (2013), pp.21-32.

[8] Gokhale UM \& Joshi YV, "A New Watermarking Algorithm Based on Image Scrambling and SVD in the Wavelet Domain", ACEEE Int. J. on Network Security, Vol.2, No.03, (2011).

[9] Chaturvedi N \& Basha S, "A Novel SVD based Digital Watermarking Scheme using DWT and A comparative study with DWT-Arnold, SVD-DCT and SVD-DFT based watermarking", International Journal of Digital Application \& Contemporary research, Vol.1, (2013).
[10] Jia SL, "A novel blind color images watermarking based on SVD", Optik-International Journal for Light and Electron Optics, Vol.125, No.12,(2014), pp.2868-2874.

[11] Thind DK \& Jindal S, "A semi blind DWT-SVD video watermarking", Procedia Computer Science, Vol.46, (2015), pp.1661-1667.

[12] Santhi V \& Arulmozhivarman P, "Hadamard transform based adaptive visible/invisible watermarking scheme for digital images", JISA, Vol.18, No.4, (2013).

[13] Ch.Harsha vardhan, Ramesh J \& ch.himabindu, "An Invisible watermarking scheme using 2- level DWT", ICRETE, (2016), pp. 59-62.

[14] Makbol NM \& Khoo BE, "Robust blind image watermarking scheme based on redundant discrete wavelet transform and singular value decomposition", AEU-International Journal of Electronics and Communications, Vol.67, No.2,(2013), pp.102-112.

[15] Gonzalez RC, Digital image Processing, pearson $3^{\text {rd }}$ edition, (2016).

[16] Kumar NA, Haribabu M \& Bindu H, "Novel image watermarking algorithm with DWT-SVD”, International Journal of Computer Applications, Vol.106, No.1,(2014). 